\title{
Status of the endangered ivory gull, Pagophila eburnea, in Greenland
}

\author{
Olivier Gilg • David Boertmann · Flemming Merkel • \\ Adrian Aebischer $\cdot$ Brigitte Sabard
}

\begin{abstract}
The ivory gull, a rare high-Arctic species whose main habitat throughout the year is sea ice, is currently listed in Greenland as 'Vulnerable', and as 'Endangered' in Canada, where the population declined by $80 \%$ in 20 years. Despite this great concern, the status of the species in Greenland has been largely unknown as it breeds in remote areas and in colonies for which population data has rarely, if at all, been collected. Combining bibliographical research, land surveys, aerial surveys and satellite tracking, we were able to identify 35 breeding sites, including 20 new ones, in North and East Greenland. Most colonies are found in North Greenland and the largest are located on islands and lowlands. The current best estimate for the size of the Greenland population is approx. 1,800 breeding birds, but the real figure is probably $>4,000$ adult birds (i.e.
\end{abstract}

O. Gilg $(\bowtie)$

Division of Population Biology,

Department of Biological and Environmental Sciences,

University of Helsinki, P.O. Box 65, 00014 Helsinki, Finland

e-mail: olivier.gilg@ libertysurf.fr

O. Gilg $\cdot$ A. Aebischer $\cdot$ B. Sabard

Groupe de Recherche en Ecologie Arctique,

16 rue de Vernot, 21440 Francheville, France

D. Boertmann $\cdot$ F. Merkel

Department of Arctic Environment,

National Environmental Research Institute,

Aarhus University, P.O. Box 358, 4000 Roskilde, Denmark

F. Merkel

Greenland Institute of Natural Resources,

P.O. Box 570, 3900 Nuuk, Greenland

\section{A. Aebischer}

Musée d'histoire naturelle Fribourg,

Chemin du Musée 6, 1700 Fribourg, Switzerland
$>2,000$ pairs) since all colonies have not yet been discovered and since only $50 \%$ or less of the breeding birds are usually present in the colonies at the time the censuses take place. Although this estimate is four to eight times higher than that previously arrived at, the species seems to be declining in the south of its Greenland breeding range, while in North Greenland the trends are unclear and unpredictable, calling for increased monitoring efforts.

Keywords Pagophila eburnea · Greenland . Endangered species $\cdot$ Satellite tracking $\cdot$ Climate change . Sea-ice

\section{Introduction}

The ivory gull (Pagophila eburnea) is one of the most remote and poorly known breeding birds in the northern hemisphere. Over its entire breeding range (Canadian Arctic, Greenland, Svalbard and Russian Arctic islands), it breeds either on inland cliffs and 'nunataks', i.e. rocky outcrops emerging from icecaps, or on high-Arctic barren islands or flatlands. The size of its entire circumpolar population is believed to range between just 8,000 and 11,500 breeding pairs (Gilchrist et al. 2008).

Because the ivory gull spends most of its life cycle on sea ice, a habitat that is currently shrinking during the summer season (Gascard 2008; Holland et al. 2006; Perovich and Richter-Menge 2009), the species could soon face a dramatic decline, at least in some parts of its breeding range. In Canada, where the status of the species has recently been updated to 'Endangered', recent studies claim that $80 \%$ of the breeding population has already been lost during the past 20 years (Gilchrist and Mallory 2005). Although its status is still under evaluation in all other 
countries holding breeding populations, this alarming situation has drawn wide attention to the species (Krajick 2003) and an international circumpolar 'Conservation Strategy and Action Plan' has recently been presented by leading seabird experts of the Arctic countries (Gilchrist et al. 2008). Despite that Greenland, where the species is considered 'Vulnerable' (Boertmann 2008a), is believed to hold a significant part of the world population of the ivory gull, published data are sparse for the country. The data only refer to a limited number of colonies and, with a few exceptions, have not been updated since the colonies were first described.

The main aims of our study, based on a review of published sources, extensive recent survey from 2003 to 2008 as well as coupled satellite monitoring and aerial surveys, were, therefore:

- to complete and compile the mapping of Greenland breeding sites of ivory gull to improve future monitoring and conservation of these sites

- to estimate the size of the breeding population of ivory gull in Greenland to update its numerical contribution to the world population and to assess past or future changes.

\section{Materials and methods}

Data used in this paper have three main origins: published sources, coupled satellite tracking and aerial surveys, and direct surveys (land and aerial).

Published data are few and far between. They are easy to locate since most biological expeditions investigating the Greenland bird fauna often used to publish a summary of their field observations in the Danish Ornithological Journal: 'Dansk Ornithologisk Forenings Tidsskrift'. Furthermore, several syntheses have already summarized past studies (Boertmann 1994; Falk et al. 1997; Gilg et al. 2003; Salomonsen 1950, 1961), and the 'Greenland seabird database' maintained by the National Environmental Research Institute (NERI) in Denmark also aims to maintain updated information on all seabird breeding sites known in Greenland (see, e.g. Boertmann 2008b).

Satellite tracking was initiated on ivory gulls in North Greenland in 2007 (Gilg et al. 2008a, 2008b) in two of the largest breeding colonies known in the country (i.e. Station Nord and Henrik Krøyer Holme; Table 1; Fig. 1). A total of 13 and 5 birds were fitted with satellite transmitters in 2007 and 2008, respectively. The primary aim of this study was to document movements, phenology and site fidelity of the species. However, since most birds tagged at Station Nord, where anthropogenic food supplies (i.e. organic wastes from the station's kitchen and leftover dog food) are abun- dant, appeared to be visitors from other colonies (Gilg et al. unpublished), satellite tracking also rapidly appeared to have great potential to locate new colonies or to confirm occupation of known colonies (all tracked birds were adults with incubation patches). To assess this assumption, we first listed all inland sites that had been visited more than ten times within a time period of more than 20 days by a single bird during the breeding season (between 1 July and 15 August 2007, and 15 June and 1 August 2008). Eight of the 11 resulting 'suspected breeding sites' could be checked by one of us (DB) during aerial surveys conducted between 30 July and 1 August 2008, i.e. during the chick rearing period (see below) in order to (1) validate the method, and (2) eventually estimate the size of the occupied colonies.

Direct surveys, on land or from the air, have been undertaken by the authors since 2003 in order to assess site fidelity and population size at most of the known breeding sites. All but a few of the breeding sites known for Greenland could be visited during the course of the two summers, 2007-2008, alone. Most of them had not been revisited since their discovery. Several new colonies were also found during our aerial surveys, both in south-east Greenland and in North Greenland.

In addition to these three main sources of information, we also located three new colonies (two ascertained and one suspected) by inquiring with individuals (i.e. geologists, geographers or mountaineers) that we knew had visited the poorly known nunatak areas of East Greenland $\left(68-70^{\circ} \mathrm{N}\right)$.

Colony sizes given in the results section relate to the 'highest number of adult birds seen' at the colonies from 1998-2008, or from 1978-2008 if no census data were available for the period 1998-2008.

\section{Results}

Breeding sites

Altogether, 35 breeding sites are currently (i.e. since 1978) known in south-east $(n=6)$, north-east $(n=1)$ and North Greenland ( $n=28$; Table 1; Fig. 1$)$. With the exception of three sites discovered in 1908 (Renskaeret; Manniche 1910), 1910 (Nakkehoved; Mikkelsen 1913; Salomonsen 1967) and 1961 (Station Nord; Salomonsen 1961), all these sites have been discovered recently $(\geq 1978)$ and from a limited number of expeditions (Bennike and Higgins 1989; Bennike and Kelly 1986; Falk et al. 1997; Gilg et al. 2003; Håkansson et al. 1981; Hjort et al. 1988; Hjort et al. 1983; Wright and Matthews 1980; present study). The majority of these sites $(n=20)$ are presented here for the first time (Fig. 1: blue circles). 
Fig. 1 New (dark dots) and formerly known (light dots) breeding sites of the ivory gull in Greenland and North Greenland (the latest region is detailed in upper panel). Dot size proportional to colony size (see legend box; uncounted sites included in the smallest class). Numbers on the map refer to colony descriptions in Table 1. Historical and unconfirmed breeding sites (see Table 1) are marked with dark and light stars, respectively

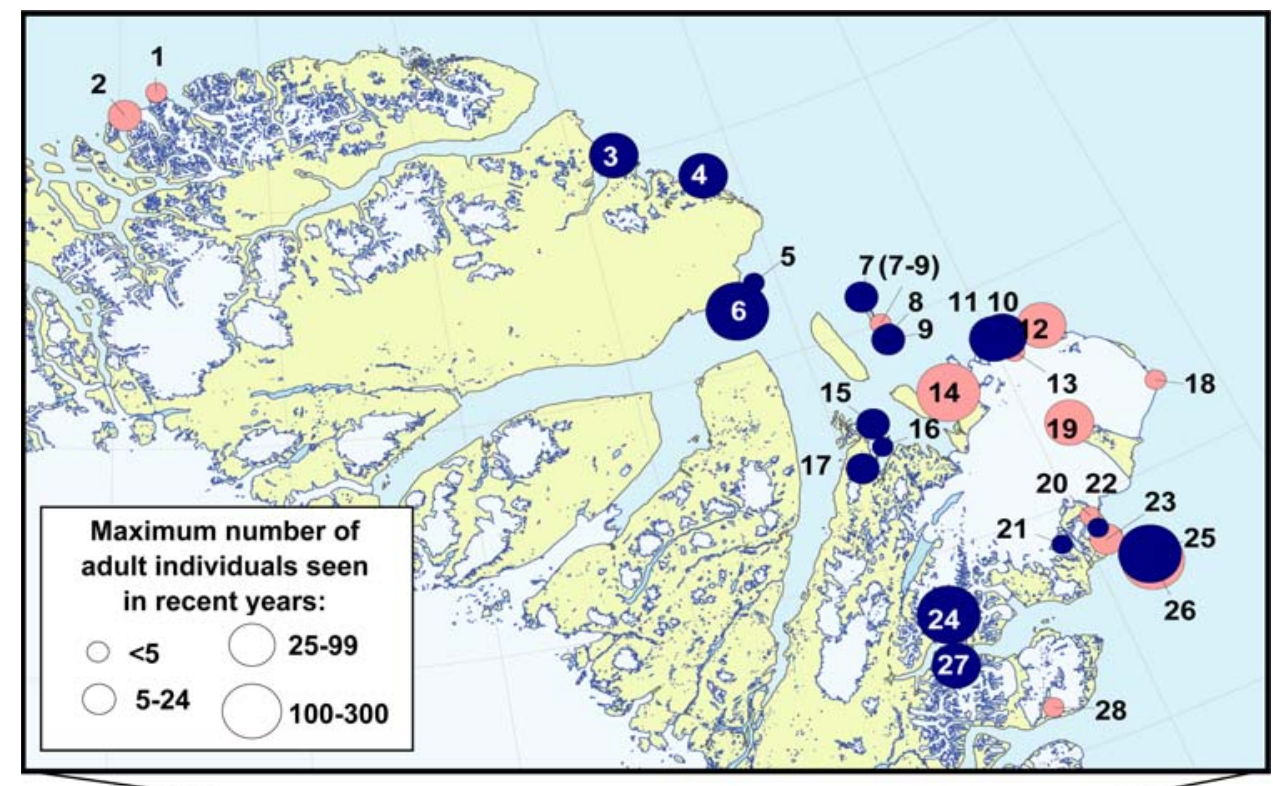

$\frac{c}{0}$
0
0
0
0
0
0
0
0
0

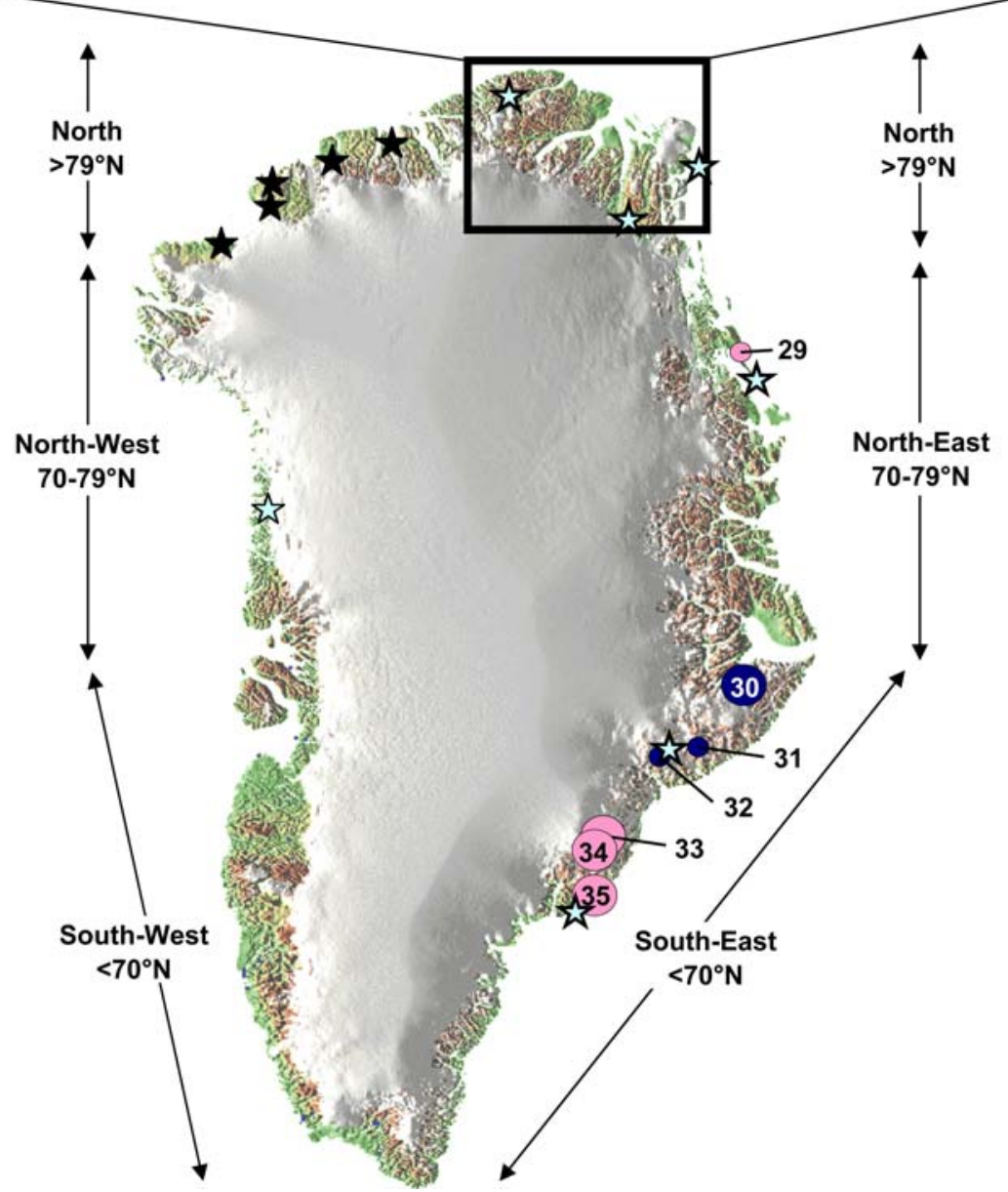

Three additional breeding sites have also been reported in north-west Greenland in the past (1854-1922; Bessels 1879; Salomonsen 1950) and six other sites are worth mentioning as possible breeding sites (Table 1; Fig. 1).

All but one of the six breeding sites found in south-east Greenland (sites \# 30-35; south of $70^{\circ}$ Lat $N$ ) are on nunataks at altitudes ranging from 1,500 to 2,500 $\mathrm{m}$ a.s.l. (site \# 35 is at approx. 1,150 $\mathrm{m}$ a.s.l.). On the other hand, all colonies found in North Greenland (sites \# 1-28) are on islands, coastal lowlands, small cliffs or on much lower nunataks (maximum altitude: $1,000 \mathrm{~m}$ a.s.l.; all but two sites below $500 \mathrm{~m}$ a.s.l.). Figure 2 presents the main types of breeding sites. 
Table 1 Ivory Gull breeding sites and numbers in Greenland

\begin{tabular}{|c|c|c|c|c|c|c|c|}
\hline Site\# & $\begin{array}{l}\text { Colony name } \\
\text { (from north to south) }\end{array}$ & Lat. $\mathrm{N}$ & Long. W & Type of site & Date & Numbers & Source \\
\hline 1 & $\begin{array}{l}\text { J. V. Jensen Land- } \\
\text { Kap Washington }\end{array}$ & c. $83^{\circ} 32^{\prime}$ & c. $38^{\circ} 42^{\prime}$ & n.d. & 4 August 1980 & "Small colony" & $\begin{array}{l}\text { Bennike and } \\
\text { Higgins (1989) }\end{array}$ \\
\hline 2 & $\begin{array}{l}\text { J. V. Jensen Land- } \\
\text { Kap Kane }\end{array}$ & $83^{\circ} 26^{\prime}$ & $39^{\circ} 48^{\prime}$ & Cliff & 1980 & c. 20 ind. & $\begin{array}{l}\text { Bennike and } \\
\text { Kelly (1986) }\end{array}$ \\
\hline 3 & $\begin{array}{l}\text { E Peary Land- } \\
\text { G.B. Schley } \\
\text { Fjord (mouth) }\end{array}$ & $83^{\circ} 01^{\prime}$ & $23^{\circ} 38^{\prime}$ & Island/lowlands & 1 August 2008 & 55 adults + chicks. & Present study \\
\hline 4 & $\begin{array}{l}\text { E Peary Land- } \\
\text { East of Kap } \\
\text { Erik Bunch }\end{array}$ & $82^{\circ} 50^{\prime}$ & $21^{\circ} 05^{\prime}$ & $\begin{array}{l}\text { Island/lowlands } \\
\text { (2 sub-colonies) }\end{array}$ & 1 August 2008 & 37 adults & Present study \\
\hline 5 & $\begin{array}{l}\text { S Peary Land-Mouth } \\
\text { Independence Fjord } 1\end{array}$ & $82^{\circ} 20^{\prime}$ & $20^{\circ} 42^{\prime}$ & Ice floe & July-August 2007 & Occupied colony ${ }^{\mathrm{d}}$ & Present study \\
\hline 6 & $\begin{array}{l}\text { S Peary Land-Mouth } \\
\text { Independence Fjord } 2\end{array}$ & $82^{\circ} 14^{\prime}$ & $21^{\circ} 27^{\prime}$ & Ice floe & $\begin{array}{l}\text { July-August } 2007 \\
\text { June } 2008 \\
1 \text { August } 2008\end{array}$ & $\begin{array}{l}\text { Probable colony }^{\mathrm{d}} \\
\text { Occupied colony }^{\mathrm{d}} \\
125 \text { adults, many pulli }\end{array}$ & $\begin{array}{l}\text { Present study } \\
\text { Present study } \\
\text { Present study }\end{array}$ \\
\hline \multirow[t]{4}{*}{7} & \multirow{4}{*}{$\begin{array}{l}\text { N Prinsesse } \\
\text { Margrethe } \emptyset \\
\text { (3 sub-colonies } \\
\text { described in 2008) }\end{array}$} & $82^{\circ} 07,8^{\prime}$ & $17^{\circ} 53,4^{\prime}$ & \multirow[t]{4}{*}{$\begin{array}{l}\text { Plateau on } \\
\quad \text { large island }\end{array}$} & 3 July 2007 & $\begin{array}{l}\text { A few breeding } \\
\text { birds seen from plane }\end{array}$ & Present study \\
\hline & & $82^{\circ} 07,8^{\prime}$ & $17^{\circ} 53,4^{\prime}$ & & 31 July 2008 & 3 pairs & Present study \\
\hline & & $82^{\circ} 08,4^{\prime}$ & $17^{\circ} 54,5^{\prime}$ & & 30 July 2008 & 6 adults, pulli seen & Present study \\
\hline & & $82^{\circ} 07,2^{\prime}$ & $17^{\circ} 52,2^{\prime}$ & & 31 July 2008 & 5 adults & Present study \\
\hline \multirow[t]{3}{*}{$7-9$} & \multirow[t]{3}{*}{$\begin{array}{l}\text { Prinsesse } \\
\quad \text { Margrethe } \emptyset \text { (overall) }\end{array}$} & \multirow[t]{3}{*}{ c. $82^{\circ}$} & \multirow[t]{3}{*}{ c. $17^{\circ} 40^{\prime}$} & \multirow[t]{3}{*}{$\begin{array}{l}\text { Plateau on } \\
\quad \text { large island }\end{array}$} & 1978 & "Colony"a & $\begin{array}{l}\text { Håkansson } \\
\text { et al. (1981) }\end{array}$ \\
\hline & & & & & $?$ & $>25$ pairs & Falk et al. (1997) \\
\hline & & & & & 27 July 2003 & No birds seen & Present study \\
\hline 8 & $\begin{array}{l}\text { SE Prinsesse } \\
\quad \text { Margrethe } \emptyset\end{array}$ & $81^{\circ} 57^{\prime}$ & $17^{\circ} 34^{\prime}$ & $\begin{array}{l}\text { Plateau on } \\
\quad \text { large island }\end{array}$ & 2007 and 2008 & Occupied colony ${ }^{\mathrm{d}}$ & Present study \\
\hline 9 & $\begin{array}{l}\text { SW Prinsesse } \\
\text { Margrethe } \emptyset\end{array}$ & $81^{\circ} 55,5^{\prime}$ & $17^{\circ} 39^{\prime}$ & Plateau on large island & 30 July 2008 & 8 pairs & Present study \\
\hline 10 & W. of Kap Prins Knud 1 & $81^{\circ} 46^{\prime}$ & $14^{\circ} 40^{\prime}$ & $\begin{array}{l}\text { Small gravel island } \\
\text { (2 sub-colonies) }\end{array}$ & $\begin{array}{l}\text { June } 2008 \\
1 \text { August } 2008\end{array}$ & $\begin{array}{l}\text { Occupied colony }{ }^{\mathrm{d}} \\
55 \text { adults }\end{array}$ & $\begin{array}{l}\text { Present study } \\
\text { Present study }\end{array}$ \\
\hline 11 & W. of Kap Prins Knud 2 & $81^{\circ} 46^{\prime}$ & $14^{\circ} 55^{\prime}$ & Small rocky island & $\begin{array}{l}\text { June } 2008 \\
30 \text { July } 2008\end{array}$ & $\begin{array}{l}\text { Occupied colony }{ }^{\mathrm{d}} \\
85 \text { adults }\end{array}$ & $\begin{array}{l}\text { Present study } \\
\text { Present study }\end{array}$ \\
\hline 12 & Nakkehoved & $81^{\circ} 44.5^{\prime}$ & $13^{\circ} 33^{\prime}$ & Cliff & $\begin{array}{l}\text { c. } 8 \text { June } 1910 \\
1978 \\
? \\
10 \text { July } 2007 \\
30 \text { July } 2008\end{array}$ & $\begin{array}{l}\text { Several nests } \\
\text { "colony"a } \\
>25 \text { pairs } \\
30 \text { ind. } \\
\text { No birds seen }\end{array}$ & $\begin{array}{l}\text { Mikkelsen (1913) } \\
\text { Håkansson et al. (1981) } \\
\text { Falk et al. (1997) } \\
\text { Present study } \\
\text { Present study }\end{array}$ \\
\hline \multirow[t]{3}{*}{13} & \multirow[t]{3}{*}{ Flade Isblink } & \multirow[t]{3}{*}{$81^{\circ} 41^{\prime} ?$} & \multirow[t]{3}{*}{$14^{\circ} 33^{\prime} ?$} & Coastal terrace & 1978 & “colony”" & $\begin{array}{l}\text { Hakansson } \\
\quad \text { et al. (1981) }\end{array}$ \\
\hline & & & & & 15 July 2007 & No birds seen & Present study \\
\hline & & & & & 30 July 2008 & No birds seen & Present study \\
\hline 14 & Station Nord & $81^{\circ} 37-38^{\prime}$ & $16^{\circ} 39-42^{\prime}$ & Coastal terrace & $\begin{array}{l}\text { June } 1961 \\
? \\
\text { 4 August } 1998\end{array}$ & $\begin{array}{l}12 \text { pairs } \\
>5 \text { pairs } \\
1 \text { pair }+1 \text { young } \\
\text { (ca. } 5 \mathrm{~km} \mathrm{~S} \\
\text { of the colony) }\end{array}$ & $\begin{array}{l}\text { Salomonsen (1961) } \\
\text { Falk et al. (1997) } \\
\text { Ko de Korte } \\
\quad \text { (pers. com. 2009) }\end{array}$ \\
\hline & & & & & End July 2001 & 20 breeding ad. + juv. & $\begin{array}{l}\text { Hans Meltofte } \\
\quad \text { (pers. com. 2003) }\end{array}$ \\
\hline & & & & & 2002 & $\begin{array}{l}140-160 \text { ad. } \\
\text { and }>40 \text { juv. }\end{array}$ & $\begin{array}{l}\text { Station staff } \\
\quad \text { (pers. com. 2002) }\end{array}$ \\
\hline & & & & & 26 July/11 Aug 2003 & c. 250 ad. and $>60$ nests & Present study \\
\hline & & & & & 20 July 2006 & $\begin{array}{l}\text { No breeding } \\
\quad(>\operatorname{lm} \text { snow } 20-7-06)\end{array}$ & $\begin{array}{l}\text { Tony Fox } \\
\quad \text { (pers. com. 2006) }\end{array}$ \\
\hline
\end{tabular}


Table 1 continued

\begin{tabular}{|c|c|c|c|c|c|c|c|}
\hline Site\# & $\begin{array}{l}\text { Colony name } \\
\text { (from north to south) }\end{array}$ & Lat. N & Long. W & Type of site & Date & Numbers & Source \\
\hline & & & & & End Aug 2006 & A few young only & $\begin{array}{l}\text { Station staff } \\
\quad \text { (pers. com. 2007) }\end{array}$ \\
\hline & & & & & 1-10 July 2007 & $\begin{array}{l}\text { c. } 60 \text { birds visiting } \\
\text { the Station }\end{array}$ & Present study \\
\hline & & & & & 12 July 2007 & $\begin{array}{l}\text { A few nest only } \\
\text { (too much snow) }\end{array}$ & Present study \\
\hline & & & & & 2008 & No birds seen & Present study \\
\hline \multirow[t]{2}{*}{15} & \multirow[t]{2}{*}{ Prins Frederik Øer ${ }^{d}$} & \multirow[t]{2}{*}{$81^{\circ} 36^{\prime}$} & \multirow[t]{2}{*}{$18^{\circ} 56^{\prime}$} & \multirow{2}{*}{$\begin{array}{r}\text { Coastal terrace } \\
\text { on peninsula }\end{array}$} & June 2008 & Occupied colony ${ }^{\mathrm{d}}$ & Present study \\
\hline & & & & & 1 August 2008 & 14 adults & Present study \\
\hline \multirow[t]{2}{*}{16} & \multirow[t]{2}{*}{ Kongefjord } & \multirow[t]{2}{*}{$81^{\circ} 30^{\prime}$} & \multirow[t]{2}{*}{$18^{\circ} 54^{\prime}$} & \multirow[t]{2}{*}{ Small island } & July 2007 & Occupied colony $^{\mathrm{d}}$ & Present study \\
\hline & & & & & 1 August 2008 & No birds seen & Present study \\
\hline \multirow[t]{2}{*}{17} & \multirow[t]{2}{*}{ Knud den Store Iskappe } & \multirow[t]{2}{*}{$81^{\circ} 26^{\prime}$} & \multirow[t]{2}{*}{$19^{\circ} 37^{\prime}$} & \multirow[t]{2}{*}{ Cliff in Canyon } & June 2008 & Occupied colony ${ }^{\mathrm{d}}$ & Present study \\
\hline & & & & & 1 August 2008 & 10 adults & Present study \\
\hline \multirow[t]{3}{*}{18} & \multirow[t]{3}{*}{ Nordostrundingen } & \multirow[t]{3}{*}{ c. $81^{\circ} 20^{\prime}$} & \multirow[t]{3}{*}{ c. $11^{\circ} 30^{\prime}$} & \multirow[t]{3}{*}{ Coastal flatlands } & 1980 & 1 old nest & Hjort et al. (1983) \\
\hline & & & & & 10 July 2007 & No birds seen & Present study \\
\hline & & & & & 1 August 2008 & No birds seen & Present study \\
\hline \multirow[t]{2}{*}{19} & \multirow[t]{2}{*}{ Kilen } & \multirow[t]{2}{*}{ c. $81^{\circ} 19^{\prime}$} & \multirow[t]{2}{*}{ c. $14^{\circ} 04^{\prime}$} & \multirow[t]{2}{*}{ Cliff } & 4 August 1980 & 75 ind. (incl. $15-20$ juv.) & Hjort et al. (1983) \\
\hline & & & & & 8 August 1985 & c. 40 ad. and $>15$ juv. & Hjort et al. (1988) \\
\hline 20 & S Antarctic Bugt & c. $80^{\circ} 55^{\prime}$ & c. $14^{\circ} 39^{\prime}$ & Cliff & 22 June 1980 & 1 pair & Hjort et al. (1983) \\
\hline 21 & W Amdrup Land & $80^{\circ} 51^{\prime}$ & $15^{\circ} 36^{\prime}$ & Small Nunatak & July-August 2007 & Occupied colony ${ }^{\mathrm{d}}$ & Present study \\
\hline 22 & E Amdrup Land & $80^{\circ} 51,5^{\prime}$ & $14^{\circ} 36^{\prime}$ & n.d. & July-August 2007 & Occupied colony ${ }^{\mathrm{d}}$ & Present study \\
\hline 23 & S Amdrup Land & c. $80^{\circ} 48^{\prime}$ & c. $14^{\circ} 32^{\prime}$ & Cliff & 10 August 1993 & c. 6 pairs & Falk et al. (1997) \\
\hline 24 & Prinsesse & $80^{\circ} 44^{\prime}$ & $18^{\circ} 54^{\prime}$ & Nunatak & July 2007 & Occupied colony ${ }^{\mathrm{d}}$ & Present study \\
\hline & Elisabeth Alper & & & & 4 July 2008 & $>200$ adults & $\begin{array}{l}\text { Fin Bo Madsen } \\
\quad \text { (pers. com. 2008) }\end{array}$ \\
\hline 25 & Henrik Krøyer & $80^{\circ} 40.3^{\prime}$ & $13^{\circ} 43.5^{\prime}$ & Large Island & 9 August 2003 & c. 150 nests & Present study \\
\hline & Holme & & & & 15 July 2007 & (>300 ad.) 10 adults & Present study \\
\hline & & & & & 29 July 2008 & No birds seen & Present study \\
\hline 26 & Henrik Krøyer & $80^{\circ} 38.5^{\prime}$ & $13^{\circ} 44^{\prime}$ & Large Island & 12 August 1980 & 10 ad. probably breeding & Hjort et al. (1983) \\
\hline & $\begin{array}{l}\text { Holme } \\
\text { (central Island) }\end{array}$ & & & $\begin{array}{l}\text { ( } 2 \text { to } 3 \\
\text { sub-colonies) }\end{array}$ & 12 July 1984 & $\begin{array}{l}\text { "Many bredding Ivory } \\
\text { Gulls, some with eggs, } \\
\text { others with young" }\end{array}$ & $\begin{array}{l}\text { "Thore" } \\
\text { (HKH Hyttebook) }\end{array}$ \\
\hline & & & & & 4/29 July 1993 & $>125$ nests (c. 510 ind.) & Falk et al. (1997) \\
\hline & & & & & 8 August 2003 & c. 135 nests (>300 ad.) & Present study \\
\hline & & & & & 12 July 2007 & $220-250$ adults $^{\mathrm{c}}$ & Present study \\
\hline & & & & & 29 July 2008 & c. 290 adults & Present study \\
\hline 27 & Caroline Mathilde Alper & $80^{\circ} 31^{\prime}$ & $19^{\circ} 11^{\prime}$ & Steep inland cliff & 1 August 2008 & 40 adults & Present study \\
\hline 28 & S Holm Land & c. $80^{\circ} 13^{\prime}$ & c. $17^{\circ} 24^{\prime}$ & Small Nunatak & 10 July 1980 & 1 pair & Hjort et al. (1983) \\
\hline 29 & Renskaeret & $76^{\circ} 41^{\prime}$ & $18^{\circ} 31^{\prime}$ & Small rocky island & 18 July 1908 & 7 pairs & Manniche (1910) \\
\hline & & & & & 1993 & 1 territorial pair & $\begin{array}{l}\text { J. Graugaard } \\
\text { (in Boertmann 1994) }\end{array}$ \\
\hline & & & & & 21 August 2004 & 1 territorial pair & Present study \\
\hline 30 & 'Hauge's Nunatak' & $69^{\circ} 45^{\prime}$ & $28^{\circ} 24^{\prime}$ & Nunatak & 1984 (beg. August?) & Several 100 s ad. $^{\mathrm{b}}$ & $\begin{array}{l}\text { Hauge Andersson } \\
\quad \text { (pers. com. 2003) }\end{array}$ \\
\hline & & & & & 26 June 2007 & 35 adults $^{\mathrm{b}}$ & Present study \\
\hline 31 & $\begin{array}{l}\text { Watkins Bjerge } \\
\quad \text { (South of point 2130) }\end{array}$ & $68^{\circ} 38^{\prime}$ & $29^{\circ} 41^{\prime}$ & Nunatak & 26 June 2008 & 2 adults & Present study \\
\hline 32 & $\begin{array}{l}\text { Lemon Bjerge } \\
\quad \text { (East of point 2460) }\end{array}$ & $68^{\circ} 30^{\prime}$ & $32^{\circ}$ & Nunatak & 1993 & At least 4 breeding birds & $\begin{array}{r}\text { Sir Chris Bonington } \\
\quad \text { (pers. com. 2004) }\end{array}$ \\
\hline & & & & & 26 June 2008 & No birds seen & Present study \\
\hline
\end{tabular}


Table 1 continued

\begin{tabular}{|c|c|c|c|c|c|c|c|}
\hline Site\# & $\begin{array}{l}\text { Colony name } \\
\text { (from north to south) }\end{array}$ & Lat. $\mathrm{N}$ & Long. W & Type of site & Date & Numbers & Source \\
\hline \multirow[t]{2}{*}{33} & \multirow[t]{2}{*}{$\begin{array}{l}\text { Kronprins Frederik } \\
\text { Bjerge Nunatak } 1\end{array}$} & \multirow[t]{2}{*}{$67^{\circ} 53^{\prime}$} & \multirow[t]{2}{*}{$34^{\circ} 25^{\prime}$} & \multirow[t]{2}{*}{ Nunatak } & 11 August 1978 & $>45$ ad. + juv. & $\begin{array}{l}\text { Wright and } \\
\text { Matthews (1980) }\end{array}$ \\
\hline & & & & & 26 June 2008 & No birds seen & Present study \\
\hline \multirow[t]{2}{*}{34} & \multirow[t]{2}{*}{$\begin{array}{l}\text { Kronprins Frederik } \\
\text { Bjerge Nunatak } 2\end{array}$} & \multirow[t]{2}{*}{$67^{\circ} 38^{\prime}$} & \multirow[t]{2}{*}{$34^{\circ} 08^{\prime}$} & \multirow[t]{2}{*}{ Nunatak } & 7 August 1978 & $30-45$ ad. +juv. & $\begin{array}{l}\text { Wright and } \\
\text { Matthews (1980) }\end{array}$ \\
\hline & & & & & 26 June 2008 & $\geq 25$ adults & Present study \\
\hline 35 & $\begin{array}{l}\text { Kronprins Frederik } \\
\text { Bjerge Nunatak } 3\end{array}$ & $66^{\circ} 48^{\prime}$ & $34^{\circ} 25^{\prime}$ & Nunatak & 30 July 1978 & $>26$ ad. + juv. & $\begin{array}{l}\text { Wright and } \\
\text { Matthews (1980) }\end{array}$ \\
\hline \multicolumn{8}{|c|}{ Historical $(<1925)$ or unconfirmed breeding sites: } \\
\hline 36 & $\begin{array}{l}\text { J. V.Jensen Land- } \\
\text { Upper Harmsworth } \\
\text { Glacier }\end{array}$ & $83^{\circ} 10-20^{\prime} ?$ & $34-36^{\circ} ?$ & n.d. & 27 July-5 August 1969 & $\begin{array}{l}16 \text { sightings at camp } \\
\text { (400 m.a.s.1.) } \\
13 \text { to } 17 \text { birds at nearby } \\
\text { Frigg Fjord (mid August) }\end{array}$ & Grant (1972) \\
\hline 37 & $\begin{array}{l}\text { Hendrik Island, } \\
\text { Warming Island } \\
\text { \& St George Fjord }\end{array}$ & c. $81^{\circ} 57^{\prime}$ & c. $53^{\circ} 15^{\prime}$ & n.d. & July 1917 & "Suspected breeding" & $\begin{array}{l}\text { Wulff } \\
\quad \text { (in Salomonsen 1950) }\end{array}$ \\
\hline 38 & Hall Land-Newman Bay & $81^{\circ} 10-55^{\prime} ?$ & $57^{\circ}-62^{\circ} ?$ & n.d. & 11/14 June 1872 & "breeding" & Bessels (1879) \\
\hline 39 & $\begin{array}{l}\text { Henrik Krøyer Holme } \\
\text { (southern island) }\end{array}$ & $81^{\circ} 38^{\prime}$ & $13^{\circ} 52^{\prime}$ & $\begin{array}{l}\text { Large } \\
\text { Island }\end{array}$ & 29 July 2008 & 1 possible breeding pair & Present study \\
\hline 40 & $\begin{array}{l}\text { Washington Land- } \\
\text { Kap Constitution }\end{array}$ & c. $80.33^{\prime}$ & c. $66^{\circ} 40^{\prime}$ & Cliff & June 1854 & 6 ad. probably breeding & $\begin{array}{l}\text { Morton } \\
\quad \text { (in Salomonsen 1950) }\end{array}$ \\
\hline 41 & $\begin{array}{l}\text { Washington Land- } \\
\text { Cass Fjord }\end{array}$ & $80^{\circ} 03^{\prime} ?$ & $64^{\circ} 30^{\prime} ?$ & Cliff & 19 June 1922 & 1 nest & $\begin{array}{l}\text { L. Koch } \\
\quad \text { (in Salomonsen 1950) }\end{array}$ \\
\hline 42 & Graeselv & $79^{\circ} 57^{\prime}$ & $24^{\circ}$ & n.d. & July 2003 & n.d. (daily sightings of birds) & Present study \\
\hline 43 & Inglefield Land & c. $79^{\circ} 05^{\prime}$ & c. $67^{\circ} 22^{\prime}$ & n.d. & $?$ & "Breeding locally" & $\begin{array}{l}\text { Rasmussen } \\
\text { and Vibe } \\
\text { (in Salomonsen 1950) }\end{array}$ \\
\hline \multirow[t]{3}{*}{44} & \multirow[t]{3}{*}{ Traekpasset } & \multirow[t]{3}{*}{$76^{\circ} 09^{\prime}$} & \multirow[t]{3}{*}{$18^{\circ} 40^{\prime}$} & \multirow[t]{3}{*}{ Cliff } & 24 July 1974 & 1 possible breeding pair & Meltofte et al. (1981) \\
\hline & & & & & $2003 \& 2004$ & $\begin{array}{l}\text { No birds seen } \\
\quad \text { (only Glaucus gulls) }\end{array}$ & Present study \\
\hline & & & & & 30 May 2008 & No birds seen & Present study \\
\hline 45 & Kippaku Island & $73^{\circ} 43^{\prime}$ & $56^{\circ} 38^{\prime}$ & n.d. & $\begin{array}{l}\text { July/August } \\
1988\end{array}$ & $\begin{array}{l}\text { Vocalizing birds } \\
(\leq 7) \text { regularly flying } \\
\text { up and down the Fjord } \\
\text { (to small Nunataks } \\
\text { 50km to the E.?) }\end{array}$ & $\begin{array}{l}\text { Peter Lyngs } \\
\quad \text { (pers. com. 2008) }\end{array}$ \\
\hline 46 & Mitivajkat & $68^{\circ} 33,5^{\prime}$ & $31^{\circ} 43^{\prime}$ & Nunatak & 1972 & "possible breeding colony" & $\begin{array}{l}\text { Wright and } \\
\text { Matthews (1980) }\end{array}$ \\
\hline 47 & KIV Steenstrups Glacier & c. $66^{\circ} 33^{\prime}$ & c. $35^{\circ}$ & n.d. & 1974 & $\begin{array}{l}\text { "strongly suspected } \\
\text { breeding site" }\end{array}$ & $\begin{array}{l}\text { Wright and } \\
\text { Matthews (1980) }\end{array}$ \\
\hline
\end{tabular}

a Colony seen from distance

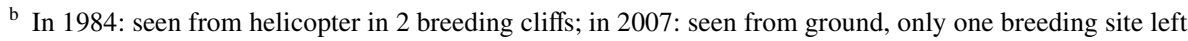

c Several clutch found between 10-15 July 2007; all but a few predated by polar bears (daily visits)

${ }^{\mathrm{d}}$ Breeding site infered from satellite monitoring (see text)

The distances between the 35 colonies and the nearest seashore vary from $<1 \mathrm{~km}$ (for the 20 "coastal" colonies) to $48 \mathrm{~km}$ (mean: $7.4 \mathrm{~km} \pm 12.5 \mathrm{SD}$ ). The colonies are located farther inland in the south (mean: $28.4 \mathrm{~km} \pm 13.2 \mathrm{SD}$ for the colonies located south of $\left.70^{\circ} \mathrm{N}\right)$, where all $(n=6)$ are on nunataks, than in the north (mean: $3.0 \mathrm{~km} \pm 6.7 \mathrm{SD}$; $n=29$ ), where most colonies (69\%) are coastal (i.e. located $<1 \mathrm{~km}$ inland) and only three are located more than $10 \mathrm{~km}$ inland. It is worth mentioning that the distance that birds from site \# 30 have to travel to reach the sea is probably
$>130 \mathrm{~km}$. Indeed, it is more likely that these birds feed on the outer Blosseville coast, where the species has regularly been reported during the breeding season over the last century (e.g. in D'Aunay Bugt and between Cape Vedel and Kangerdlugssuak: Amdrup 1902; Degerbøl and Møhl-Hansen 1935; Gilg et al. 2005; Glahder 1995; present study), than in the Scoresby Sund Fjord system, where some birds are regularly seen at the mouth of the fjord during migration (a few can also be seen feeding at glacier fronts in autumn) but rarely in the inner fjord system in summer 
Fig. 2 Different types of breeding sites used by the ivory gull in Greenland: a large (i.e. $>1 \mathrm{~km}$ wide) flat island (site \# 26), b ice floe covered with gravel (site \# 6), c small coastal lowlands/ islands ( site \# 4), $\mathbf{d}$ coastal gravel terrace (site \# 14), e small inland cliff below 1,000 m.a.s.l. (site \# 17) and $\mathbf{f}$ steep inland nunatak above 1,000 m.a.s.l. (site \# 30; photos: O.G. adf and D.B. bce)
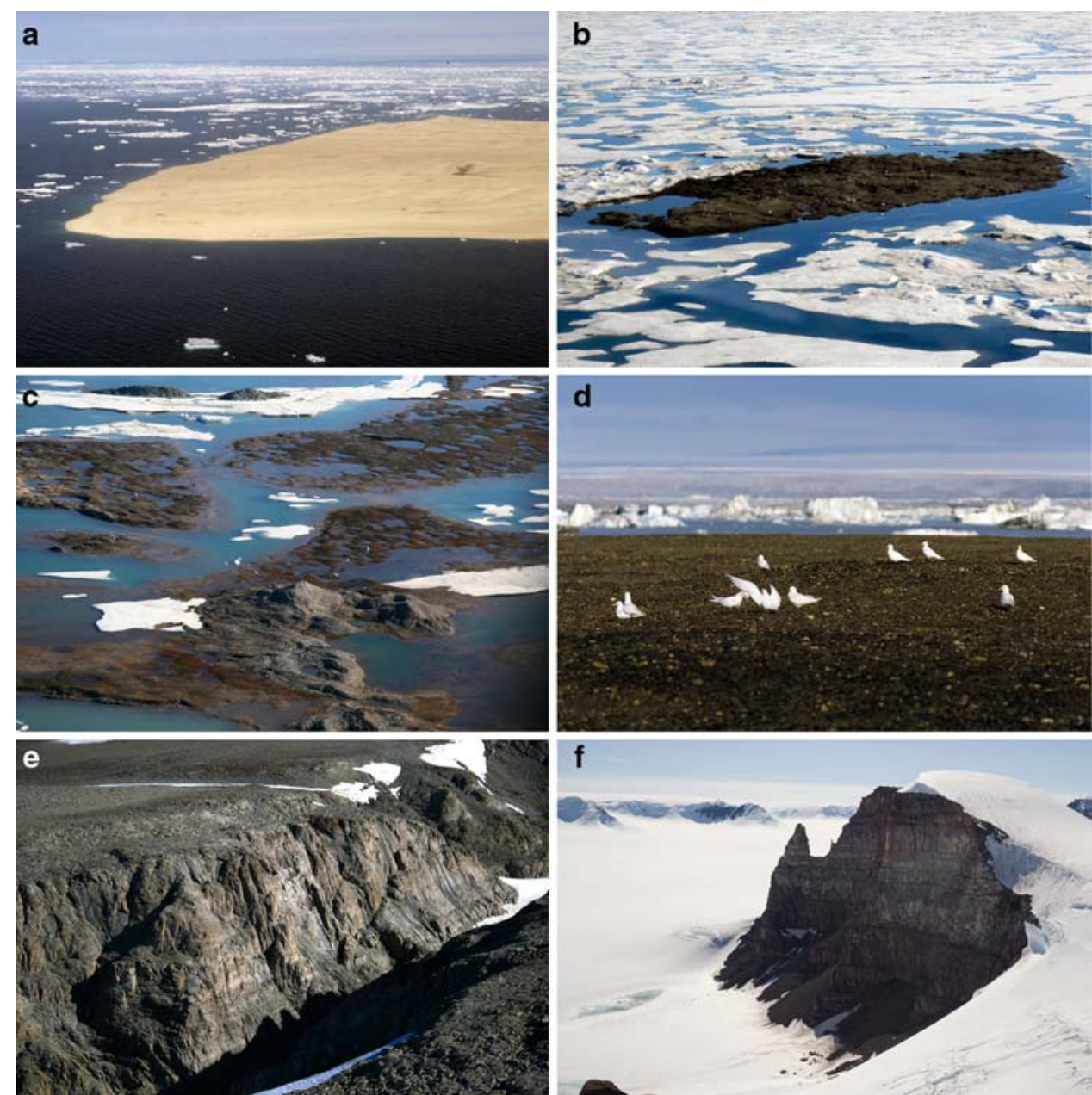

(see, e.g. Gilg et al. 2005; Hørring 1939; Meltofte 1976b; Pedersen 1930; Petersen 1941). Even though such large distances between the colonies and the nearest feeding grounds appear important, they are not exceptional for the species, as shown by the long distances regularly travelled by coastal breeding ivory gulls monitored by satellite tracking in North Greenland (regularly more than $200 \mathrm{~km}$ per foraging bouts; Gilg et al. unpublished).

At the bottom of Table 1, we also present the historical or suspected breeding sites that need to be confirmed before they can be considered as current breeding sites. A few additional sites have not been considered in this table, despite that some authors have suggested they could hold breeding birds. For example, on the islets of Rosio off Cape Marie Valdemar, Manniche (1910; see also Meltofte 1975) suggested possible breeding activity, but we failed to find any birds at this location during an aerial survey in 2007. The numerous icebound islands between Skaerfjorden, Jøkelbugten and Nioghalvfjerdsfjorden $\left(77^{\circ} 30^{\prime}\right.$ to $\left.79^{\circ} 40^{\prime} \mathrm{N}\right)$ are also looking very favourable for the breeding of ivory gull but the few biologists that have visited the area have not found any breeding sites to date (C. Bay in Boertmann
1994; Cabot et al. 1988; Bennike 2007). In addition, there are some old doubtful reports from Hochstetter Foreland (Løppenthin 1932), Hold with Hope (Boyd 1935), and Mallemukfjeld (Koch and Bertelsen in Manniche 1910).

Finally, it must be mentioned that at least three deserted colonies (former confirmed or suspected breeding sites) are currently used by other gull species. At Traekpasset (site \# 44; Table 1), glaucous gulls (Larus hyperboreus) are nowadays regularly breeding on the cliff where Meltofte et al. (1981) suspected breeding of one pair of ivory gulls in 1974 (Gilg et al. 2003, 2005). On the eastern side of Renskaeret Island (site \# 29; Table 1), where Manniche (1910) found a colony in 1908, black-legged kittiwakes (Rissa tridactyla) currently breed on the only available small cliff (Gilg et al. 2005). At Mallemukfjeld, where Koch and Bertelsen (in Manniche 1910) reportedly found 50 ivory gulls in 1907 (but see above), there are large colonies of black-legged kittiwake, northern fulmar (Fulmarus glacialis) and glaucous gulls (Falk and Møller 1997; Hjort et al. 1983; Manniche 1910). Hence, it cannot be ruled out that a number of historical colonies of ivory gull, especially coastal colonies between $75^{\circ} \mathrm{N}$ and $80^{\circ} \mathrm{N}$, have been deserted in Greenland 
during the twentieth century due to inter-specific competition.

\section{Colony sizes}

Colony sizes reported from Greenland ranged from one or a few pairs (several sites) to several hundred individuals (max. 125-150 nests and 300-510 adults on Henrik Krøyer Holme in 1993 and 2003). The number of breeding birds found in a colony can vary greatly from year to year. At Station Nord, for example, colony size varied from a few pairs $(2001,2006$, 2007) to $150-250$ breeding birds $(2002,2003)$. Very late snow cover (at Station Nord in 2006-2007) and systematic nest predation (by polar bears at Henrik Krøyer Holme in 2007) were the most likely causes of fluctuation at these sites. Non-breeding (in 2008 compared to 2007) could not be explained at two other sites where limitation due to snow cover (site \# 16) or predation (site \# 12) was unlikely.

If the colonies are sorted according to the 'highest number of adult birds seen' (see "Methods"), then 4 (i.e. 15\%) out of the 26 breeding sites with positive counts (i.e. excluding 9 sites that are known but not censused) have less than 5 birds, 6 sites (23\%) have between 5 and 24 birds, 10 sites (42\%) between 25 and 99 birds and 5 sites (19\%) between 100 and 300 birds (Table 1; Fig. 1). The average colony size is 69 birds ( $\pm 90 \mathrm{SD}$ ) but most birds are found in large colonies since the median is only 33 birds. Median colony size was significantly greater in islands/coastal lowland colonies than on inland cliffs/nunataks $(U=38$ : $P<0.05$, Mann-Whitney $U$ test).

\section{Population size}

Given the above-mentioned inter-annual fluctuation, and since most colonies have only been counted once since their discovery, the overall population estimate for Greenland must be considered with caution. If we sum the highest colony sizes reported for the 26 colonies that have ever been counted (i.e. we have known coordinates for nine colonies that have never been censused), we reach a total of approx. 1,800 adult birds, i.e. more than twice the population size previously estimated for Greenland (Gilchrist et al. 2008).

This number may be overestimated by double counts if we assume that some adult birds can change breeding sites on a yearly basis. However, if we sum the mean number of adult birds counted at each of these colonies (i.e. 1,336 birds for 26 colonies) instead of the maximum, and then extrapolate this result to all 35 colonies (to take into account the nine colonies that have been located but never counted), we also end up with a total of approx. 1,800 birds.

In addition, the count of 850 adult birds in 2003 at the three largest breeding sites and the total of 970 adults

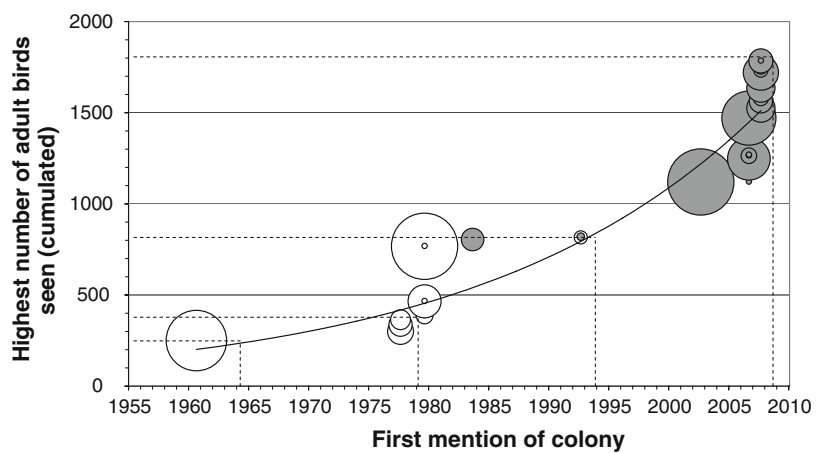

Fig. 3 Contribution of newly discovered breeding sites to our knowledge of the ivory gull population size in Greenland. Each circle presents a single colony, positioned (on the $x$ axis) according to the date of its discovery, and with a radius proportional to the "highest number of adult birds seen' at this site in recent years (see Sects. "Methods" and "Results"; grey circles for colonies first described in this paper). The $y$-value of any given colony is the sum of the "highest number of adult birds seen' for all colonies that were known at that time. e.g. if no new colony had been found since 1964,1979 or 1994, our best estimate for the size of the Greenland ivory gull population would only be 250, 364 or 820 adult birds, respectively, while it is currently close to 1,800 (i.e. roughly a twofold increase every 15 years; see dashed lines). The trend is exponential (given by the grey line)

reported in 2008 from 23 breeding sites (i.e. only two-thirds of the known colonies), excluding the three largest, also supports the view that previous published figures have considerably underestimated the current Greenland population.

\section{Discussion}

Our results, coupling satellite telemetry and direct surveys, were unexpectedly performing to locate new breeding sites. In 2008 only, the monitoring of six 'transient' birds (i.e. that did not breed at Station Nord where they were trapped) led to the discovery of six new colonies. More than half of the colonies, representing more than half of the current population size estimate, have been discovered within the last 5 years (2003-2008; Table 1; Fig. 3). Does this mean that the population is now satisfactorily known or, on the contrary, that many colonies are still unknown?

What is the real population size in Greenland?

In the results section, based on the number of adult birds attending colonies, we reported that the population size of Greenland ivory gulls was potentially as high as 1,800 adult birds $( \pm 25 \%)$, i.e. twice as high as previously estimated (Gilchrist et al. 2008). It is likely, moreover, that this should be considered a minimum.

First, because the 'breeding population' we are attempting to assess should also consider, in addition to the breeding adults censused at the breeding sites, the failed breeders 
(some of which resume their breeding cycle and are overlooked at the time of the censuses) and possibly also a fraction of the large number of non-breeding adults reported for the species in some years at the breeding sites or offshore (Gilg et al. 2005; Joiris 1996; Tomkovich 1986; Volkov and Korte 2000). Indeed, if we acknowledge the fact that for ivory gulls non-breeding is an important but stochastic process resulting from extrinsic factors (e.g. local snow or ice cover, mass predation, etc.) and affecting entire colonies (rather than related to the individual condition of the birds), then it would be theoretically relevant to include these 'non-breeding' birds in an estimate of the overall breeding population estimate, since they clearly belong to the pool of potentially reproducing birds. This, however, remains a theoretical concern (probably relevant for other colonial breeders as well) since we have no data to test this bias at this time. It must be mentioned, however, that if interannual site fidelity (or breeding philopatry) is high in ivory gulls, as strongly suggested by Volkov and Korte (2000), then our 'highest number of birds seen' at each colony (see "Methods") includes some of these birds, thereby reducing this bias.

Second, not all breeding birds attend their nests or young when the censuses take place (Volkov and Korte 2000). In fact, earlier studies have shown that for the ivory gull, the number of breeding adults reported from single censuses of a colony (e.g. all Greenland census presented in Table 1) is close to the total number of breeding pairs using the colony, hence advocating the use of a two-fold correction factor (Mallory et al. 2008; Robertson et al. 2007; H. Strøm, personal communication 2009). This low attendance rate is also evident from our satellite tracking data: the breeding birds we monitored in 2007, presumably mainly males, only spent approx. $50 \%$ or less of their time at the colony sites during the breeding period (Gilg et al. unpublished). Furthermore, even when both partners are present at the colony one is often resting or grooming on the ice within a few hundred metres of the nest, and therefore overlooked during the census.

Last, from our recent discovery of several new colonies, it can be assumed that several additional colonies in the remotest parts of Greenland have yet to be reported. Single birds seen in the north along frozen fjords during the breeding season (e.g. Jørgen Brønlund Fjord, Meltofte 1976a) are not particularly helpful in relation to the discovery of new colonies, since our satellite tracking results show regular movements of breeding birds over drift ice or fast ice at distances of more than $100 \mathrm{~km}$ from the colonies. On the other hand, based on our satellite tracking, ivory gulls almost never fly inland in summer, except to reach their colonies (during spring migration, some birds also cross the icecap, as indicated by 6-8 adult birds seen flying eastwards on 29 May 2006 at $67^{\circ} 28^{\prime} 32^{\prime \prime} \mathrm{N} / 41^{\circ} 28^{\prime} 23^{\prime \prime} \mathrm{W}$, i.e. $175 \mathrm{~km}$ from the nearest nunatak; Johannes Lang, personal communication 2006). Therefore, based on repeated observations of birds flying inland during the breeding season, additional colonies are highly likely in North (e.g. sites \# 36 and \# 42) and north-west Greenland (site \# 44). Finally, the recently discovered offshore island of Tobias $\varnothing\left(79^{\circ} 20^{\prime} \mathrm{N}, 15^{\circ} 48^{\prime} \mathrm{W}\right.$; Bennike et al. 2006; Mohr and Forsberg 2001) should be checked carefully for ivory gulls in the future, since unidentified gulls have been reported here and due to its location (near the offshore ice edge) and flat topography, ivory gulls could potentially breed at this site.

Due to the above reasons, the estimate of 1,800 adult birds for Greenland is probably far below the true figure. It is not unrealistic to consider, for example, that non-attending breeding birds (the second source of underestimation presented above) are at least as numerous as the attending breeding birds counted at the colonies. Hence, the total population for Greenland could easily be $>4,000$ breeding adults (or $>2,000$ breeding pairs), i.e. four to eight times more than previously estimated (Gilchrist et al. 2008).

\section{Population trends}

The increase in the estimate for the Greenland population (see above) is mainly due to the recent (2003-2008) discovery of new colonies. If we had only summed the colony sizes (including those from recent censuses) reported for colonies discovered prior to 1990 , then the estimated population size would just be approx. 800 birds (Fig. 3). Therefore, the apparent increasing trend shown in Fig. 3 cannot be used to support any true population change. This figure will probably continue to increase in the future, since it is likely that additional new colonies will be discovered.

Periodic non-breeding, a common feature in Arctic birds (Bertram and Roberts 1934; Bird and Bird 1940; Marshall 1952), including the ivory gull (Gilchrist et al. 2008; Robertson et al. 2007; Volkov and Korte 2000), is another obstacle to assess past and future population trends. Indeed, it is difficult to assess the true population trends in ivory gull colonies from the year of discovery because, by definition, the initial census is always positive, while subsequent censuses can be positive or null. This shortcoming can be overcome by (1) removing the first census (discovery) or (2) removing all subsequent zero counts. Since often only two censuses were available for a given colony for the present study, removing the first count was not a viable option; therefore, the second option was used in preparation of Table 2 .

Until 2000, all but a few of the Greenland colonies had only been counted once and no reliable population trends could be presented (Boertmann 1994; Gilchrist et al. 2008). During the course of 2003-2008, we managed to visit most of the colonies that had previously been censused. Only five 
Table 2 Ivory gull population trends inferred from positive multiple counts at eight breeding sites

\begin{tabular}{|c|c|c|c|c|c|}
\hline \multirow[t]{2}{*}{ Site \# } & \multirow[t]{2}{*}{ Colony name (from north to south) } & \multicolumn{4}{|c|}{ Period of reference } \\
\hline & & $<1978-2008$ & 1978-2008 & 1988-2008 & 1998-2008 \\
\hline 12 & Nakkehoved & & & & - \\
\hline 14 & Station Nord & + & & & \pm \\
\hline 19 & Kilen & & - & & \\
\hline 25 & Henrik Krøyer Holme (northern Island) & & & & - \\
\hline 26 & Henrik Krøyer Holme (central Island) & & + & - & $=$ \\
\hline 29 & Renskaeret & - & & & \\
\hline 30 & 'Hauge’s Nunatak’ & & - & & \\
\hline 34 & Kronprins Frederik Bjerge Nunatak 2 & & - & & \\
\hline \multicolumn{2}{|c|}{ Main population trend for the periods: } & $?(n=2)$ & $\downarrow(n=4)$ & $\downarrow(n=1)$ & $\downarrow(n=4)$ \\
\hline
\end{tabular}

colonies (sites \# 2, 35, 20, 23, 28), with earlier reports of limited numbers of breeding birds ( 20 and 26 birds; 1,6 and 1 pairs, respectively), could not be checked. The trends are presented qualitatively (Table 2) for the eight colonies with accurate and positive multiple counts (details in Table 1). Empirically, Table 2 shows a dominant declining trend since 1978. This trend is particularly clear in southeast Greenland, where apparent declines or extinctions are reported for all monitored colonies (sites \# 29-35; Table 1), including a dramatic ( $>80 \%)$ decline at the largest known colony in this region (site \# 30) from 1984 to 2007 (declined from several $100 \mathrm{~s}$ of adults to only 35) and a 35\% decline in a smaller colony (site \# 34) from 1978 to 2008. The situation is more ambiguous in North Greenland where some colonies possibly have declined while others have increased over the long term, including two of the largest since their discovery in 1961 and 1980 (sites \# 14 and \# 26, respectively). Given the year-to-year variation in colony size (see above) and the limited data set, any further statistical analysis of the overall population trend would be of little confidence.

\section{Scenarios for future population trends in Greenland}

Although repeated counts that can be used to assess population trends are rare in Greenland, the available data seem to indicate that ivory gulls have declined in the southern colonies (south of $70^{\circ} \mathrm{N}$ ), while trends in the north are unclear. The likely decline in southern colonies can be compared with recent trends reported for Canadian colonies (Gilchrist and Mallory 2005; Mallory et al. 2003; Robertson et al. 2007). In Canada, the $80 \%$ decline documented over a period of 20 years has been hypothesized to have resulted mainly from pollution and excessive hunting (e.g. in NW Greenland; Braune et al. 2006, 2007; Gilchrist and Mallory 2005). Because gulls declined over their entire Canadian breeding range, including very different habitats types and regions, it has been suggested that these two negative factors act mainly during the winter period or migration. Indeed, post-breeding Canadian birds regularly move to West Greenland and especially the Thule area (Renaud and McLaren 1982), where hunting occurs. However, since all ivory gull populations probably use the same wintering grounds (i.e. the north-western part of the Atlantic Ocean; Strøm et al. unpublished), while only the Canadian population declined at such a dramatic speed (Gilchrist et al. 2008), we suggest that alteration of habitat (mainly the sea ice) in Northern Canada during summer and the migration period could also be a cause of the observed decline.

In any case, according to our current knowledge, it is difficult to forecast future changes in population sizes in Greenland or elsewhere. If the summer sea ice, and especially the ice edge, continues to move away from Svalbard and Franz-Joseph Land at the current speed (see, e.g. recent summer trends at http://www.seaice.de), it will be difficult for the species to continue to breed in these Arctic archipelagos. In North Greenland, summer sea ice should remain for a much longer period (ACIA 2005; Overland and Wang 2007). As a result, the population from North Greenland may decline in future in synchrony with other populations if it is limited by unfavourable conditions during the winter or migration periods, or remain stable, or even increase as a result of e.g. immigration of birds from Svalbard and northwestern Russian colonies if it is the extent and quality of summer sea ice that drives the population dynamics of the species. We believe, moreover, that the recent discovery of several new colonies in North Greenland is best explained by previous poor knowledge rather an increase in population size. However, future colonization of new sites should also be considered as a likely scenario in North Greenland. Especially, the coastal lowlands and small islands of eastern Peary Land (where sites \# 3 and \# 4 where discovered in 2008; see also Fig. 2c) and Johannes V. Jensen Land (including a very favourable area in Bliss Bay which was 
surveyed for the species, unsuccessfully, in 2007; Gilg et al. 2008b) should be regularly monitored in the future to confirm or deny colonization.

Ivory gulls were probably among the first vertebrates to colonize North Greenland after the last ice edge. At least, they were already present approx. 4,000 years ago, according to remains found and dated in Eskimo settlements of Jørgen Brønlund Fjord (Grønnow and Jensen 2003). Today, their future is in jeopardy, with changes in the extent and quality of sea ice in summer being one of the main threats. The challenge this species will have to face in years to come is to adapt (see, e.g. Forcada et al. 2008) to this changing environment rapidly, or to relocate to more suitable breeding areas. Because North Greenland can be regarded as the last region in the Arctic where sea ice will remain in summer in the future (ACIA 2005), it might well also become the last region where this species may manage to breed. The current size of the regional population could even increase in the near future due to immigration of birds from Svalbard or north-western Russia (see above). Evidence of such long-distance relocation is still lacking (mark-recapture programs are in progress in Greenland, Svalbard and Russia but are hard to implement on a circumpolar scale and in such remote regions), but the recent discovery of 'offshore' colonies (i.e. birds breeding on ephemeral ice floes; Boertmann et al. 2010) in North Greenland is, at least, positive evidence that ivory gulls can relocate to locally more favourable breeding areas in the short term.

As the ivory gull could well be the first vertebrate species this century to become extinct as a consequence of human-induced climate change and the associated decline in summer sea ice (Gilg et al. 2008a), its main and almost exclusive habitat, Arctic biologists and conservation agencies should prioritize protection of its breeding areas (i.e. breeding sites and related feeding grounds) and regular monitoring of the species in North Greenland.

Acknowledgments We are very grateful to Freddy Mariaux, Luc Hardy, Benoît Sittler, Pierre Leguesdron, Vladimir Gilg, Kent Olsen and Lars Maltha Rasmussen who helped in the field, to captain Leif Petersen who navigated the aircraft in 2008, to Carey Smith, Hallvard Strøm, Maria Gavrilo, Andrei Volkov, Hans Meltofte and Niels Martin Schmidt who commented and improved previous drafts of the manuscript, to Ko de Korte, Hans Meltofte, Tony Fox, Fin Bo Madsen, Hauge Andersson, Sir Chris Bonington, Johannes Lang, Eckart Håkansson and Peter Lyngs for providing unpublished data, to John Lau Hansen (Greenland Command), the military staff of Station Nord, the Sirius Sledge Patrol, the Greenland Home Rule, the Danish Polar Center, Polog, Norlandair (formerly Air Iceland), Kirsten Fadnæs Eriksen, Nette Levermann, Kristbjörg Björnsdóttir, Hauge Andersson, Aka Lynge, Jan Almqvist, Sebastian Ravn Rasmussen, David Smari Johannsson and Friðrik Adolfsson who helped with the permits and the logistics. Funding and equipments were provided by the National Geographic Society, Prix GORE-TEX initiative, Fondation Avenir Finance, the Bureau of Minerals and Petroleum, Greenland Home
Rule, the Arctic Ocean Diversity Census of Marine Life Project, Magasins Intermarché, Société Henry Maire, Lestra, MSR, GREA, F. Paulsen and other contributors.

\section{References}

ACIA (2005) Arctic climate impact assessment. Cambridge University Press, Cambridge

Amdrup G (1902) Carlsbergfondets Expedition Til Øst Grønland 1898-1900. Part 1: Beretning om Expeditionen. Medd Gronl 27:1-372

Bennike O (2007) Notable bird observations in North-east Greenland, 1992-1998. Dansk Orn Foren Tidsskr 101:24-26

Bennike O, Higgins AK (1989) Some notable bird records from North Greenland, 1979-1986. Dansk Orn Foren Tidsskr 83:87-88

Bennike O, Kelly M (1986) Bird observations in central North Greenland, 1984. Dansk Orn Foren Tidsskr 80:29-34

Bennike O, Mikkelsen N, Forsberg R, Hedenäs L (2006) Tuppiap Qeqertaa (Tobias Island): a newly discovered island off northeast Greenland. Polar Rec (Gr Brit) 42:309-314. doi:10.1017/ S003224740600550X

Bertram DL, Roberts BB (1934) Notes on East Greenland Birds, with a discussion of the Periodic Non-breeding among Arctic Birds. Ibis 13:816-831. doi:10.1111/j.1474-919X.1934.tb01346.x

Bessels E (1879) Die Amerikanische Nordpol-Expedition. Wilhelm Engelmann, Leipzig

Bird CG, Bird EG (1940) Some remarks on non-breeding in the Arctic, especially in North-east Greenland. Ibis 14:671-678. doi:10. 1111/j.1474-919X.1940.tb01680.x

Boertmann D (1994) A annotated checklist to the birds of Greenland. Medd Gronl Biosci 38:1-63

Boertmann D (2008a) Grønlands Rødliste-2007 (The Greenland RedList). Greenland Home Rule Government \& Danmarks Miljøundersøgelser, Nuuk

Boertmann D (2008b) The lesser black-backed gull, Larus fuscus, in Greenland. Arctic 61:129-133

Boertmann D, Olsen K, Gilg O (2010) Ivory gull colony on ice floe. Polar Rec (Gr Brit) 46(236) (in press)

Boyd LA (1935) The Fiord Region of East Greenland. Special Publication $\mathrm{N}^{\circ}$ 18. Am Geogr Soc, New York

Braune BM, Mallory ML, Gilchrist HG (2006) Elevated mercury levels in a declining population of ivory gulls in the Canadian Arctic. Mar Pollut Bull 52:978-982. doi:10.1016/j.marpolbul.2006.04.013

Braune BM, Mallory ML, Gilchrist HG, Letcher RJ, Drouillard KG (2007) Levels and trends of organochlorines and brominated flame retardants in Ivory Gull eggs from the Canadian Arctic, 1976 to 2004. Sci Total Environ 378:403-417. doi:10.1016/j.scitotenv.2007.03.003

Cabot D, Goodwillie R, Viney M (1988) Irish expedition of North-East Greenland 1987. Report of the expedition based in North Sondermarken and South Nordmarken $\left(77^{\circ} 40^{\prime} \mathrm{N} 20^{\circ} 51^{\prime} \mathrm{W}\right) 28$ May-13 August 1987. Barnacle Books, Dublin

Degerbøl M, Møhl-Hansen U (1935) Birds (Aves). Medd Gronl 104:1-30

Falk K, Møller S (1997) Breeding ecology of the fulmar Fulmarus glacialis and the Kittiwake Rissa tridactyla in high-arctic northeastern Greenland, 1993. Ibis 139:270-281. doi:10.1111/j.1474-919X. 1997.tb04625.x

Falk K, Hjort C, Andreasen C et al (1997) Seabirds utilizing the Northeast Water polynya. J Mar Syst 10:47-65. doi:10.1016/S09247963(96)00066-8

Forcada J, Trathan PN, Murphy EJ (2008) Life history buffering in Antarctic mammals and birds against changing patterns of climate and environmental variation. Glob Change Biol 14:2473-2488 
Gascard JC (2008) Exploring arctic transpolar drift during dramatic sea ice retreat. EOS 89:21-23. doi:10.1029/2008EO030001

Gilchrist HG, Mallory ML (2005) Declines in abundance and distribution of the ivory gull (Pagophila eburnea) in Arctic Canada. Biol Conserv 121:303-309. doi:10.1016/j.biocon.2004.04.021

Gilchrist G, Strøm H, Gavrilo MV, Mosbech A (2008) International Ivory Gull conservation strategy and action plan. CAFF Technical Report No. 18. Conservation of Arctic Flora and Fauna (CAFF) International Secretariat, Circumpolar Seabird Group (CBird), Akureyri

Gilg O, Sabard B, Sittler B, Mariaux F, Leguesdron P, Gilg V (2003) Ecopolaris 2003. Ecological expedition to North Greenland and Dove Bugt: North-East Greenland National Park. Field report. GREA, Francheville

Gilg O, Sabard B, Kampp K et al (2005) Ecopolaris-Tara 5 expedition to NE Greenland 2004. GREA, Francheville

Gilg O, Aebischer A, Sabard B (2008a) Ismågen - det 21. århundredes Dronte? (Ivory Gull: the XXI ${ }^{\text {st }}$ century Dodo?). Dansk Orn Foren Tidsskr 102:307-308

Gilg O, Sabard B, Aebischer A, Hardy L, Bernard F (2008b) Ecopolaris \& Sagax expeditions to E \& N Greenland 2007. Preliminary field report. GREA, Francheville

Glahder C (1995) Hunting in Kangerlussuaq, East Greenland 19511991. An assessment of local knowledge. Medd Gronl Man Soc 19:1-86

Grønnow B, Jensen JF (2003) The Northernmost Ruins on the Globe. Eigil Knuth's archaeological investigations in Peary Land and adjacent areas of high Arctic Greenland. Medd Gronl Man Soc 29:1-403

Håkansson E, Bennike O, Mølgaard P, Frykman P (1981) Norgrønlandske fugleobservationer-Somrene 1976 og 1978. Dansk Orn Foren Tidsskr 75

Hjort C, Hakansson E, Stemmerik L (1983) Bird observations around the Nordøstvandet polynya, Northeast Greenland, 1980. Dansk Orn Foren Tidsskr 77:107-114

Hjort C, Hakansson E, Mølgaard P (1988) Bird observations on Kilen, northeasternmost Greenland, 1985. Dansk Orn Foren Tidsskr 82:19-24

Holland MM, Bitz CM, Tremblay B (2006) Future abrupt reductions in the summer Arctic sea ice. Geophys Res Lett 33:1-5. doi:10.1029/2006GL028024

Hørring R (1939) Birds. 6. og 7. Thule expedition til Sydøstgrønland 1931-33. Medd Gronl 108:1-44

Joiris CR (1996) At-sea distribution of seabirds and marine mammals around Svalbard, summer 1991. Polar Biol 16:423-429. doi:10.1007/BF02390424

Krajick K (2003) In search of the ivory gull. Science 301:1840-1841. doi:10.1126/science.301.5641.1840

Løppenthin B (1932) Die Vögel Nordostgrönlands zwischen $73^{\circ} 00^{\prime}$ und $75^{\circ} 30^{\prime} \mathrm{N}$. Br Medd Gronl 91:1-127

Mallory ML, Gilchrist HG, Fontaine AJ, Akearok JA (2003) Local ecological knowledge of ivory gull declines in Arctic Canada. Arctic 56:293-298

Mallory ML, Stenhouse IJ, Gilchrist G, Robertson G, Haney JC, Macdonald SD (2008) Ivory Gull (Pagophila eburnea). In: Poole A (ed) The Birds of North America online, Cornell Lab of Ornithology,
Ithaca. Available via DIALOG. http://bna.birds.cornel.edu/bna/ species/175 (doi:10.2173/bna.175). Accessed 15 Feb 2009

Manniche ALV (1910) The terrestrial mammals and birds of northeast Greenland. Medd Gronl 45:1-199

Marshall AJ (1952) Non-breeding among arctic birds. Ibis 94:310 332. doi:10.1111/j.1474-919X.1952.tb01821.x

Meltofte H (1975) Ornithological observations in Northeast Greenland between $76^{\circ} 00^{\prime}$ and $78^{\circ} 00^{\prime} \mathrm{N}$. Lat. 1969-71. Medd Gronl 191:172

Meltofte H (1976a) Ornithological observations in Southern Peary Land, North Greenland, 1973. Medd Gronl 205:1-57

Meltofte H (1976b) Ornithologiske observationer i Scoresbysundomradet, Østgrønland, 1974. Dansk Orn Foren Tidsskr 70:107-122

Meltofte H, Elander M, Hjort C (1981) Ornithological observations in Northeast Greenland between $74^{\circ} 30^{\prime}$ and $76^{\circ} 00^{\prime}$ N. lat., 1976. Medd Gronl Biosci 3:1-56

Mikkelsen E (1913) Perdus dans l'Arctique. Récit de l'expédition de "l'Alabama" 1909 à 1912. Mame, Tours

Mohr JJ, Forsberg R (2001) Searching for new islands in sea ice. Nature 416:35. doi:10.1038/416035a

Overland JE, Wang M (2007) Future regional Arctic sea ice declines. Geophys Res Lett 34:L17705. doi:10.1029/2007GL030808

Pedersen A (1930) Fortgesetzte Beiträge zur Kenntnis der Säugetierund Vogelfauna der Ostküste Grönlands. Medd Gronl 77:341507

Perovich DK, Richter-Menge JA (2009) Loss of sea ice in the Arctic. Ann Rev Mar Sci 1:417-441. doi:10.1146/annurev.marine. 010908.163805

Petersen J (1941) Dagbogsoptegnelser om Fuglenes Forkomst ved Scoresbysund Station fra Efteraaret 1934 til Sommeren 1935. Dansk Orn Foren Tidsskr 35:123-125

Renaud WE, McLaren PL (1982) Ivory Gull (Pagophila eburnea) Distribution in Late Summer and autumn in Eastern Lancaster Sound and Western Baffin-Bay. Arctic 35:141-148

Robertson GJ, Gilchrist HG, Mallory ML (2007) Colony dynamics and persistence of Ivory Gull Breeding in Canada. Avian Cons Ecol 2:1-18

Salomonsen F (1950) The Birds of Greenland. Ejnar Munksgaard, Copenhagen

Salomonsen F (1961) Ismagen (Pagophila eburnea (Phipps)) som ynglefugl i Grønland. Dansk Orn Foren Tidsskr 55:177-180

Salomonsen F (1967) Fuglene på Grønland. Rhodos, Copenhagen

Tomkovich PS (1986) Materialy po biologii beloi chaiki na ostrove Graem Bell (Zemlya Frantsa Iosifa) (in Russian: Data about the biology of the Ivory Gull on Graham Bell Island; Franz Josef Land). In: Ilichev VD (ed) Aktualnye problemy ornithologii (Actual issues of ornithology). Nauka, Moscow, pp 34-49

Volkov AE, Korte Jd (2000) Breeding ecology of the ivory gulls Pagophila eburnea in Sedova Archipelago, Severnaya Zemlya. In: Ebbinge BS, Mazourov YL, Tomkovich PS (eds) Heritage of the Russian Arctic. Research, conservation and international cooperation, Ecopros Publishers, Moscow, pp 483-500

Wright NJR, Matthews DW (1980) New nesting colonies of the Ivory Gull Pagophila eburnea in southern East Greenland. Dansk Orn Foren Tidsskr 74:59-64 